\title{
Endplate Deformation Due to Open and Strutted Intervertebral Devices
}

\author{
ANTONIO VALDEVIT, PhD, ${ }^{1}$ ANNA KEDZIERSKA, MS, ${ }^{2}$ MICHELLE B. GALLAGHER, MS, ${ }^{3}$ \\ JENNIFER M. SCHNEIDER, MS, ${ }^{3}$ PETER F. ULLRICH, MD ${ }^{3}$ \\ ${ }^{1}$ Health Sciences Practice Group, SEA Limited, Columbus, Ohio, ${ }^{2}$ Department of Biomedical Engineering, Stevens Institute of Technology, Hoboken, New Jersey, \\ ${ }^{3}$ Titan Spine LLC, Mequon, Wisconsin
}

\begin{abstract}
Background: There is an absence of work on vertebral endplate response to peripheral loading following disc removal and interbody placement. Endplate deflection into the interbody space may impart beneficial strain on the developing fusion mass, influencing bone formation and remodeling. The aim of this study was to verify endplate deformation due to peripheral loading using a custom transducer and to investigate whether endplate motion is inhibited by implant design.

Methods: A total of 14 porcine (L4, L5) vertebrae were assigned to open or strutted implant designs. A custom transducer was placed on the endplate while $500 \mathrm{~N}$ was applied to the implant at $1 \mathrm{~Hz}$ for 500 cycles. Endplate motion was acquired for each time point and averaged among specimens of the same design. The rates and magnitudes of endplate deformation were compared between implant designs using unpaired $t$ tests.

Results: Peripheral loading of both implant designs resulted in endplate deflection into the interbody space. The open implant design demonstrated an increased rate and magnitude of endplate deformation when compared with strutted implants.

Conclusion: Interbody cage design directly influences the dynamic motion of the vertebral endplate during cyclic loading. A larger, faster deflection of the endplate could increase the strain rate, duration, and magnitude on the developing interbody fusion mass. These parameters of dynamic strain have been correlated with increased bone formation and remodeling.

Clinical Relevance: Unimpeded endplate deformation in an open cage design could impart a strain pattern on the developing fusion mass that increases bone formation and remodeling, ultimately leading to a faster and stronger fusion.
\end{abstract}

Biomechanics

Keywords: endplate deformation, implant, dynamic strain, dynamic bone remodeling, osteogenesis

\section{INTRODUCTION}

Interbody cage selection plays a key role in the success of a spinal fusion surgery. In a 2014 study by Rajaee et $\mathrm{al}^{1}$ reviewing data from the Nationwide Inpatient Sample, the primary diagnosis in $40 \%$ to $47 \%$ of spinal fusion revision surgeries was "a mechanical complication of the orthopaedic device, implant or graft." The main function of an interbody cage is to correct spinal deformities, provide stability, and ensure an optimal chemical and mechanical environment for arthrodesis to occur. $^{2}$ Differences in cage material and design affect subsidence resistance, construct stability, and fusion rate, all factors that influence the success rate of the fusion surgery.

Mechanical load sharing between the interbody device and the graft is one factor shown to influence the fusion rate. ${ }^{2-4}$ Stress shielding is a well-described mechanical phenomenon that often occurs when a stiff device is implanted in series with bone. A reduction in the stiffness of interbody fusion cages has been shown to enhance fusion in an animal model at 6 months and 3 years. ${ }^{2,4}$ Whereas it is well known that the material properties such as modulus of elasticity can affect the stiffness of the cage, the effective stiffness results from a combination of the material properties and cage design. ${ }^{5}$ One approach to modifying the cage stiffness is decreasing the amount of material in the implant by creating a porous construct. With the adoption of additive manufacturing, this approach is becoming more common. Still, the level of stress shielding involves more than just matching the stiffness of the implanted materials; it is also important to minimize 
the influence of the cage on the natural physiologic response of the vertebral body and endplate.

When the intervertebral disc is removed and replaced by an interbody cage, the endplate is exposed to a combination of bone graft and implant material. The result is an effective stiffening of the endplate and a shift in the normal physiological load transfer that may lead to undesired remodeling and resorption of bone. ${ }^{6}$ Kanayama et $\mathrm{al}^{7}$ investigated the effects of 11 different lumbar interbody cages on intracage pressure during loading. They found a correlation between the intracage pressure and the largest central aperture size and concluded that if 2 different cages have equivalent total porous surface area, then the cage with the largest central aperture produces less stress shielding than those with several smaller pores, regardless of cage material. Their ${ }^{7}$ hypothesis was that the larger pore allowed for more deflection of the representative graft material into the cage, though the movement of the endplate was not directly measured. We are not aware of any studies that have directly measured the movement of the endplate during implant loading.

Endplate deformation in an intact functional spinal unit has been measured in prior studies. ${ }^{8,9}$ Results demonstrate a flexible endplate that bends into the vertebral body as a response to pressure from the intervertebral disc. Maximum deformation occurs at the center of the endplate and has been observed to average approximately $0.3 \mathrm{~mm}$ of movement. ${ }^{9}$ However, removal of the pressure imposed by the intervertebral disc could result in a change to the endplate movement direction. Speculation on the basis of the structure of the vertebra would suggest endplate movement into the interbody space. The vertebral body has a strong cortical outer shell, a core consisting of weaker cancellous bone and interosseous fluid, and a flexible endplate with limited permeability to fluid flow. ${ }^{10-12}$ It is hypothesized that under peripheral loading the combination of the increased internal pressure in conjunction with a mechanically weak central vertebral region and a stiff outer shell predisposes the endplate to external deflection.

Performing comparative evaluations regarding the interaction of interbody devices with the vertebral endplate under in vitro conditions is a complicated undertaking and has traditionally been restricted to the field of computational mechanical simulations. ${ }^{13,14}$ In this study, we examined the effects of anterior lumbar interbody fusion (ALIF) implant design upon the micromovement of the vertebral endplate during cyclic peripheral loading using a custom transducer. More specifically, the purposes of the investigation were to (1) verify endplate expansion into the interbody space during peripheral loading of the vertebra and (2) examine the differences in endplate micromotion of ALIF devices made with identical material and comparable commercial footprint with and without a central support strut. We hypothesized that open implants would facilitate unimpeded endplate motion and result in increased endplate expansion as compared with an implant equipped with a central strut (Figure 1).

\section{MATERIALS AND METHODS}

Two ALIF implants with commercial footprints were fabricated from the same Titanium-6Aluminum-4Vanadium alloy to minimize experimental variables. Commercially, the device representing an open design (No Strut, Figure 2, left) is made from titanium alloy, whereas the device representing a strutted device (Figure 2, right) is made from polyetheretherketone (PEEK). Titanium was chosen for this study because the material properties of PEEK limit the design, requiring a strut to provide sufficient mechanical integrity under cyclic dynamic loading. The endplate contact area was similar between designs, with $428 \mathrm{~mm}^{2}$ for the open device and $408 \mathrm{~mm}^{2}$ to $505 \mathrm{~mm}^{2}$ for the strutted device, ranging from no-strut to full-strut contact. Both devices were of sufficient size to engage the vertebral periphery for loading. Porcine specimens were selected for this study. A porcine vertebra is slightly smaller in geometry and displays a flatter endplate than a human vertebral body. However, the advantages of uniformity with respect to geometry and strength are significant as compared with the large variability associated with human cadaveric specimens. A total of $14 \mathrm{~L} 4$ or L5 porcine vertebrae were excised and cleaned of excess soft tissue (100$\mathrm{kg}$ animals, Animal Technologies, Tyler, Texas). Cartilaginous material was removed from the superior endplate per the manufacturer's recommended surgical procedure. It has been shown biomechanically that as little as $1 \mathrm{~mm}$ of endplate removal can result in a significant loss of mechanical integrity. ${ }^{15}$ The cartilaginous layer was also removed from the inferior vertebral endplates to facilitate good adhesion between the specimen and the embedding material. Specimens were embedded 
Device with CentralStrut in A-P Direction

Open or No Strut Device
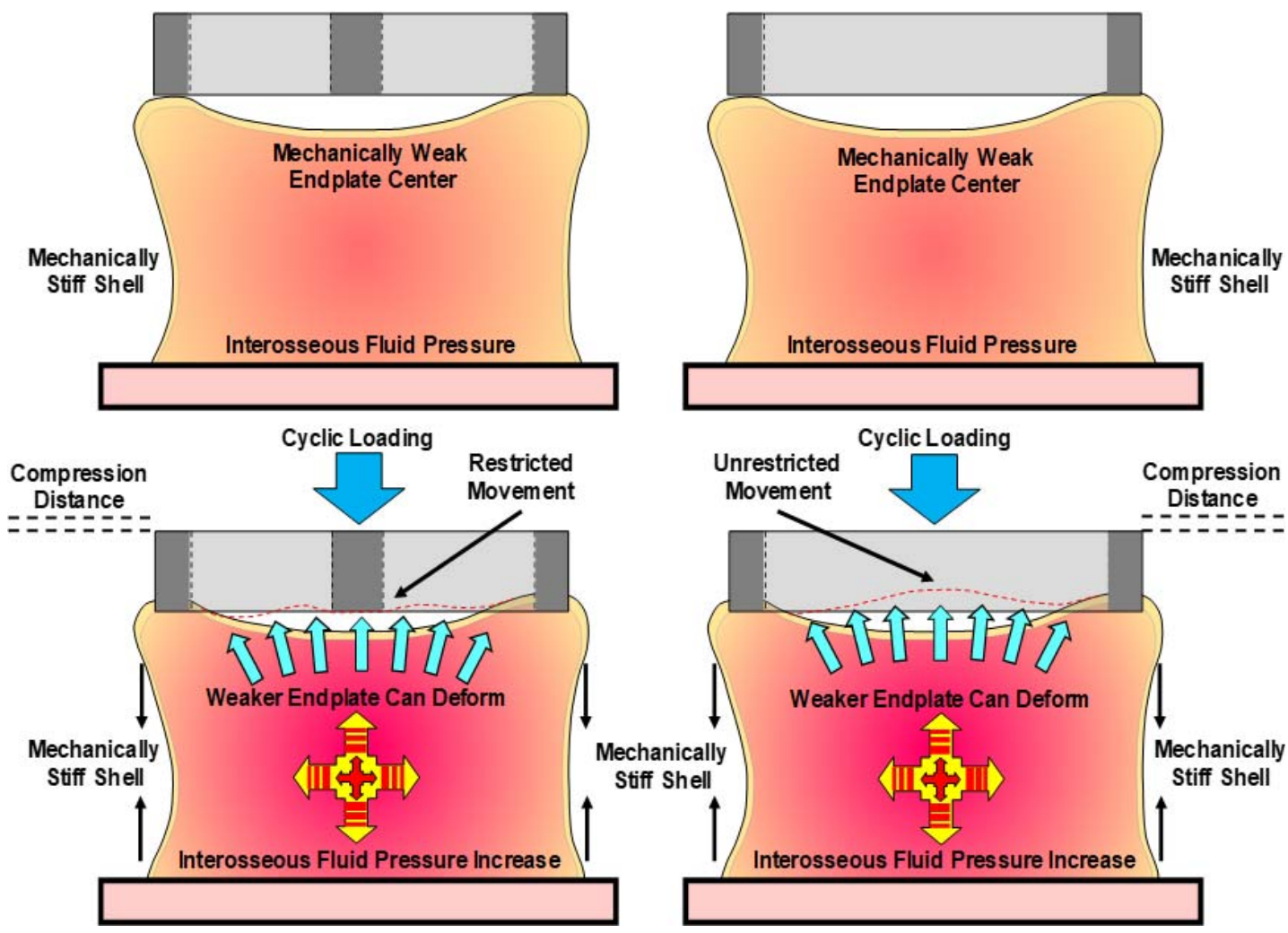

Figure 1. Schematic representation of hypothesized endplate deformation due to implant loading along the vertebral periphery of open and strutted cage designs.

in resin (Bondo, 3M, St Paul, Minnesota) to facilitate positioning within the testing machine. Specimens were randomly but equally assigned to the open or strutted implant group.

A custom-designed strain gauge-based displacement transducer was fabricated and calibrated to record endplate movement under cyclic loading of the vertebral periphery. The transducer records changes in displacement through a strain gauge mounted at the apex of a flexible central arc. When the relative separation distance between the tabs of the transducer is altered due to tension or compression, a corresponding increase or decrease in the arc

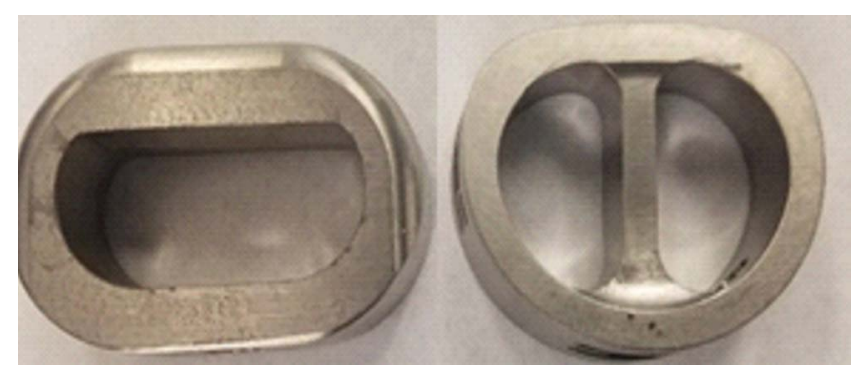

Figure 2. Left-No strut implant design (ie, open implant). Right-Implant design with a central strut (ie, strutted implant). diameter is generated and results in the deformation of the strain gauge located at the apex of the arc. ${ }^{16}$ Using a Wheatstone bridge circuit and amplifier, the gauge resistance change is transformed to an output voltage that is subsequently converted to a known displacement using a previously generated calibration curve between transducer output voltage and the change in tab-separation distance. To ensure the performance of the transducer, a pretest recording under the initial low load compression value and posttest recording under the upper load compression value were evaluated. The output fluctuation during a static measurement was a maximum of only $3 \%$ for both pretest and posttest recording. Prior to use, the transducer and accompanying electronics were allowed to equilibrate in the laboratory environment for a minimum of 20 minutes. This combined with complete thawing of the test specimens before setup permitted electrical and thermal stabilization of the strain gauge and associated electronics. To reproducibly locate the transducer upon the vertebral surface in a consistent manner applicable to both implant designs, a location was labeled with a set anterior-posterior 


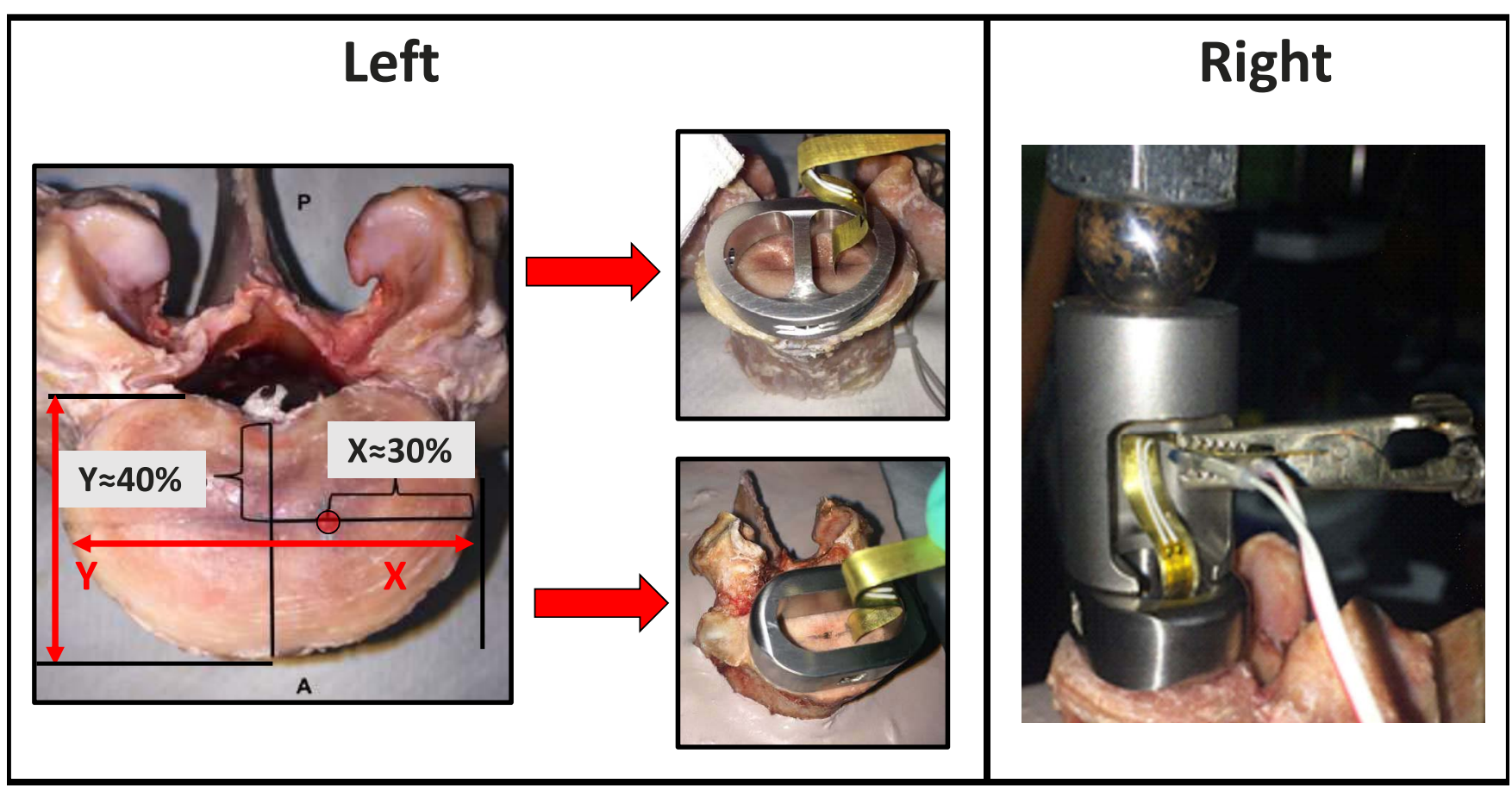

Figure 3. Experimental set up to measure endplate bulging during cyclic loading.

(AP) and medial-lateral (ML) distance (see Figure 3, left). The rationale for the ML location selection criterion was that for implants incorporating a central strut design, location of the transducer could not be placed at the midpoint of the ML vertebral distance due to the presence of the strut. Selection of the $70 \%$ ML and $40 \%$ AP locations facilitated consistent transducer positioning for both designs, thereby permitting comparison of relative endplate micromotion effects due to the presence of the strut without influence due to regional variations.

To record endplate movement under periphery loading, 1 tab of the displacement transducer was secured to a spring-loaded alligator clip affixed to a magnetic-based manipulator arm, whereas the other tab was used to sense endplate expansion (Figure 3, right).

The multiple degrees of freedom associated with the arm facilitated positioning of the transducer upon the vertebral endplate surface while a custom fixture interfaced with the actuator of the testing machine to apply a compressive force upon the implant. Prior to loading, the transducer was manually brought into contact with the vertebral endplate at the identified location. Contact was verified by observing a change in the voltage output of the transducer, indicating deformation of the flexible arc due to vertebral endplate contact. The voltage output of the transducer was nulled prior to loading. To account for variable alignment resulting from implant contact with the vertebral surface under cyclic testing, a spherical stainless-steel ball was placed between the actuator of the testing machine and the fixture in contact with the implant. Such a procedure facilitated a more uniform load transfer between the loading axis of the testing machine, the implant, and the underlying vertebral endplate. Experimental studies involving endplate loading often involve compression or combinations of loading modalities for bending. These studies are traditionally conducted under single-cycle nondestructive static or load-to-failure conditions. In the context of the present study, destructive loading would certainly manifest endplate involvement, though static, single-cycle failure loading is not representative of physiological biomechanical conditions. Furthermore, in the case of single static subfailure load application, the endplate response could not be characterized under repetitive loads, which represent a more clinically relevant response scenario. The aim of the study is to determine whether ALIF device design plays a role in the fusion process via endplate interaction under cyclic subfailure loading.

In the present study, a cyclic compressive load of $500 \mathrm{~N}$ was applied upon the respective implants at a rate of $1 \mathrm{~Hz}$ for 500 cycles by a testing machine (TA Instruments, ElectroForce 3300, Eden Prairie, Min- 


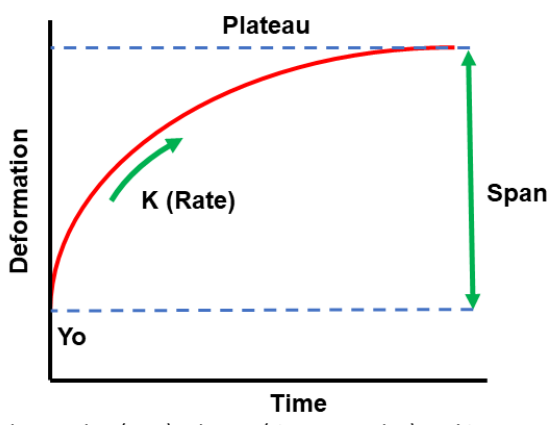

- Yo is the $Y$ value $(\mathrm{mm})$ when $X$ (time or cycles) and is expressed in the same units as $Y(\mathrm{~mm})$

- Plateau is the $Y$ value at infinite time or cycle number

- $\mathrm{K}$ is the rate constant expressed in reciprocal of the $\mathrm{X}$ value $\left(\mathrm{s}^{-1}\right.$ or cycles $\left.{ }^{-1}\right)$

- Half-Life is computed as $\ln 2 / K$ and represents the point at which Yo has changed by $50 \%$. It is expressed in the same units as the $X$ values (time or cycle number)

- Span is defined as the difference between Yo and the Plateau (total difference between the start and completion of the test). It is expressed in the same units as Yo $(\mathrm{mm})$

Figure 4. Graphical representation of an exponential function used for fitting of endplate and vertebra-implant deformation data under cyclic loading.

nesota). This fatigue-loading condition has been shown to manifest implant subsidence and/or endplate engagement yet does not contribute to significant specimen degradation because the testing time duration is on the order of several minutes. ${ }^{5,17,18}$ Continuous data for applied load and overall deformation of the vertebra-implant combination was acquired for loading cycle 10 and at subsequent 25-cycle intervals thereafter. Simultaneously, the applied load of the testing machine and resulting endplate deformation data from the transducer was continuously recorded at $40 \mathrm{~Hz}$ by a data acquisition system (DI-145, Dataq Instruments, Akron, Ohio) receiving a priori calibrated input signals from the testing machine and strain gauge amplifier (gain $=$ 500X). No amplification or signal conditioning was required for the load signal from the testing machine.

The transducer deformation data at each time point were averaged for the respective implants and

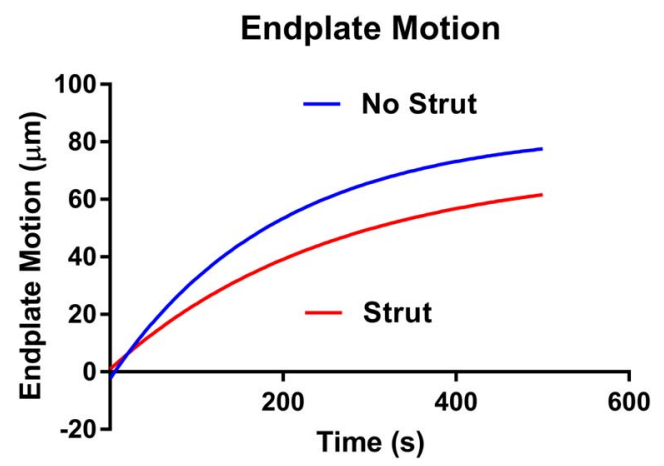

Figure 5. Fitted exponential curves to the open and strutted implant endplate motion.

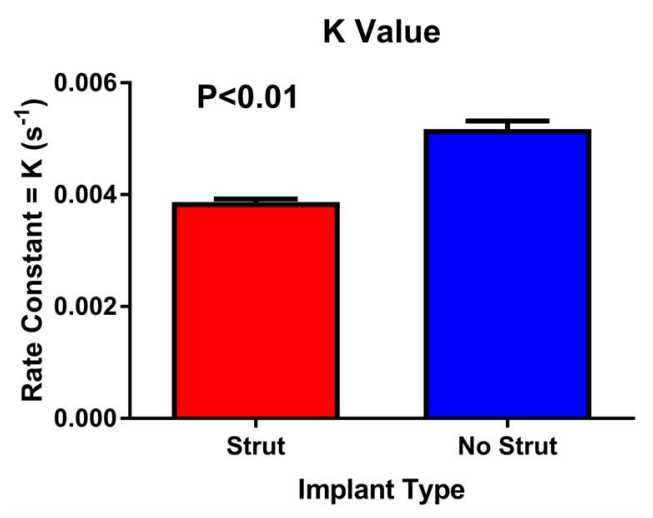

Figure 6. Resulting $\mathrm{K}$ value from the exponential regression for implants with and without a central support strut.

subjected to a nonlinear exponential regression of endplate deformation versus time (Prism 5.0, GraphPad Inc, San Diego, California). The regression equation used for fitting is represented in Figure 4.

This mathematical description has proven effective in describing bone-implant interaction performance under cyclic loading. ${ }^{5}$ The respective parameters from the regression analyses associated with the endplate deformation attributed to strutted and open (no strut) implants were compared using an unpaired Student $t$ test with significance $\alpha$ set to $P<.05$. To compute an experimental sample size, the expected standard deviation of each group was placed at 0.01 $\mathrm{mm}($ or $10 \mu \mathrm{m})$ at a significance level of $\alpha=.05$ (2tailed). The distance represents a distance significantly greater than the resolution of the transducer. An experiment consisting of a sample size of 7 in each group has $90 \%$ power to detect a difference between means of $0.019 \mathrm{~mm}$ with a significance level $\alpha=.05$ (2-tailed; N Stat, GraphPad).

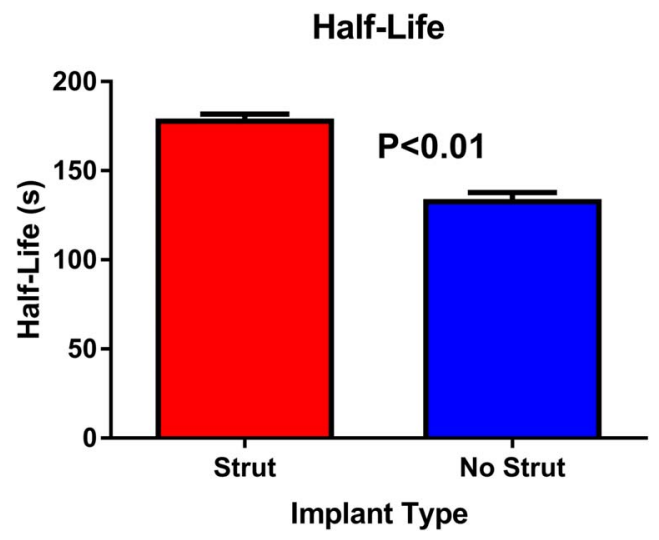

Figure 7. Resulting half-life calculation from the exponential regression for implants with and without a central support strut. 
Expansion Change (Span)

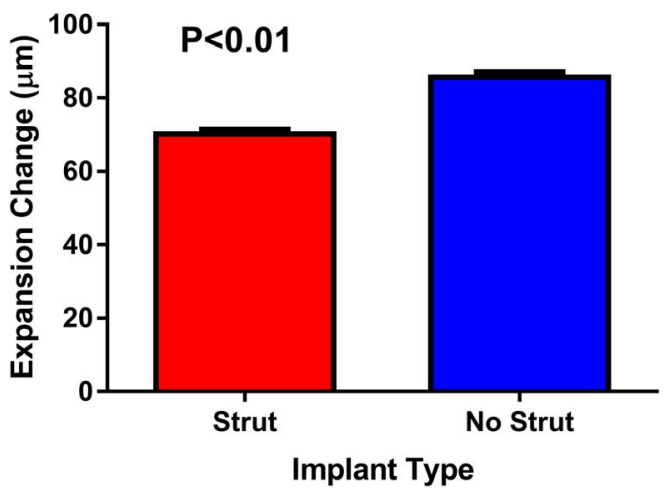

Figure 8. Resulting span calculation from the exponential regression for implants with and without a central support strut.

\section{RESULTS}

The results of cyclic loading upon the implant/ vertebra structure were represented by exponential function for both implant designs, similar to those observed in previous studies conducted by this laboratory. ${ }^{18}$ The compressive change of the implant-vertebra structure was below $10 \mu \mathrm{m}$ for both devices, indicating the results for endplate deformation were from movement of the vertebral endplate, not from endplate penetration of the cage (ie, subsidence). Both the open and strutted implants demonstrated a single exponential fit for endplate deformation (Figure 5).

The resulting rate (ie, $\mathrm{K}$ value) of endplate deformation between the 2 implants is seen in Figure 6. A statistically increased rate of endplate deflection $(P<.01)$ was seen for open implants compared with implants possessing a central strut. The result of more rapid endplate expansion for open devices was reciprocated with respect to the half-life comparison (Figure 7) where open devices manifested a reduced number of cycles $(P<.01)$ to achieve the half-life definition of a $50 \%$ change in increased endplate expansion from loading initialization relative to strutted devices.

In examining the compressive change or span parameter, implants lacking a central support strut displayed a statistical increase in endplate motion at the completion of cyclic loading $(P<.01$; Figure 8$)$. More specifically, the increase in endplate motion due to endplate peripheral loading through open devices was greater than devices with a central support strut by approximately $15.4 \pm 0.1 \mu \mathrm{m}$.

\section{DISCUSSION}

Studies examining the motion of the endplate have been limited to intact functional spinal units. ${ }^{8,9}$ To date, no experimental studies have been reported that directly measure endplate micromotion during implant loading. The goal of this study was to verify and record the movement of the central endplate during cyclic, nondestructive dynamic testing. The study further investigated whether ALIF device design would affect endplate deformation during dynamic subfailure loading, indicating the potential for differences in bone formation and remodeling between designs. Two implants were fabricated from identical material, with 1 implant displaying a hollow core and the other possessing a central strut. Both implants were subjected to cyclic loading while endplate motion was recorded using a custom transducer. An illustration of the theorized endplate motion is seen in Figure 1.

The results of this study confirm endplate deformation during peripheral implant loading and demonstrate a direction of that motion into the interbody cage. As such, in a complete spinal unit the combined deformation of both endplates would compress, thus inducing mechanical strain on the contents within the interbody device. This supports the hypothesis of Kanayama et $\mathrm{al}^{7}$ that the higher intracage pressure observed for the large continuous pore design is a result of more deflection into the graft space. Following initial healing and partial replacement of graft material by woven bone, the developing fusion mass becomes mechanically functional and can remodel as a response to mechanical strain. ${ }^{19}$ Because the woven bone of the developing fusion mass is more pliable than the vertebral endplate, ${ }^{20}$ it can be inferred that the compression would decrease the effective bone volume within the cage, inducing strain on the developing fusion mass. Bone formation and remodeling in the interbody space are similar to those found in the development of growing bone ${ }^{2}$ and in long bone healing. ${ }^{20}$. Previous studies have demonstrated that in vivo bone formation is unaffected by static loads ${ }^{21,22}$ but is significantly influenced by dynamic loading. ${ }^{22,23}$ The effect of dynamic loading on bone is based on the rate, duration, and magnitude of the applied strain. ${ }^{24}$ The dynamic outcome parameters evaluated in this study can provide insight into the influence of cage design on these strain factors. 
The rate constant of endplate deformation (Figure 6) was significantly greater for the open cage design than the strutted implant. Therefore, one would deduce that the rate of the applied strain on the developing fusion mass in an open cage would be greater than the strutted design. Duncan et $\mathrm{al}^{24}$ demonstrated that a greater rate of change in applied strain on bone increased the resulting bone formation. This observation has been replicated several times in vivo. ${ }^{21,22}$ In addition, the derived parameter half-life (Figure 7) indicates that implants without a central support require significantly less time to achieve the asymptotic limit or maximum amount of endplate deformation. As such one would conclude that the strain from maximum endplate deformation is observed for a longer period of time for the open cage than the strutted design. The literature indicates that an increased duration of applied strain leads to increased bone formation. ${ }^{23,25}$ With an increased rate and longer duration of applied strain, the open cage design would be expected to have an increased amount of bone formation. In vivo testing demonstrates that greater bone formation leads to a faster fusion ultimately resulting in a more stable construct. Van Dijk et $\mathrm{al}^{4}$ compared cages with the same design but different materials in a Dutch milk-goat model. They hypothesized the cage with reduced stiffness would increase the strain experience by the bone graft within the cage, enhancing the mechanical stimulation and lead to increased rate of fusion. After 6 months the cage with lower stiffness showed a significantly increased rate of interbody fusion, confirming their hypothesis. ${ }^{4}$ Kandziora et $\mathrm{al}^{3}$ compared cages of the same material but different designs in a sheep model and found the device with a larger central opening demonstrated an accelerated fusion. They attributed the increased fusion rate to the design of the cage, concluding the open cage lead to lower stressshielding on the incorporated bone graft and as such led to a higher interbody fusion mass found in the cage. In addition, this group evaluated stability of the construct and correlated the faster fusion to a significantly higher biomechanical stiffness.

In addition to the rate and duration, the design of the cage significantly influenced the resulting span of endplate motion, as seen in Figure 8. The span represents the maximum endplate deformation observed in the cycles complete for this study (approximately 8 minutes) and is indicative of the expected deformation per cycle during longer loading durations ( $>8$ minutes) because the data trends toward an asymptotic limit. A larger endplate deformation would imply a greater magnitude of strain on the developing fusion mass. Implants without a central support provided 15.4 $\pm 0.1 \mu \mathrm{m}$ of additional endplate expansion over the course of the test than did strutted devices. An increase in the magnitude of strain has demonstrate multiple benefits for bone growth. It is well known and accepted that bone mass increases with an increase in mechanical strain ${ }^{25}$ and bone resorption is a consequence of immobilization, weightlessness, and stress-shielding. In addition, Frankenburg et $\mathrm{al}^{26}$ demonstrated a correlation between the magnitude of loading on bone substitute material and the rate of remodeling. In a canine model, bone cement in areas exposed to higher loads was resorbed and replaced by host cortical bone more rapidly than cement in areas of low loading. Moreover, the development of either woven or lamellar bone, which impacts the mechanical stiffness and strength of the bone, ${ }^{2}$ is directly related to the magnitude of applied strain. ${ }^{27}$ Smit et $\mathrm{al}^{2}$ found that the trabecular architecture (ie, structure and density) within a spinal cage changes during the fusion process, and this process is influenced by the magnitude of strain on the developing fusion mass. They found ${ }^{2}$ that cages that allow the fusion area to receive higher magnitudes of strain demonstrated a more homogeneous and mature fusion indicated by higher trabecular thickness and trabecular spacing.

Furthermore, the specific amount of increased endplate expansion demonstrated by the open cage may play a role in the remodeling process. Bone healing induced by implant placement consists of two distinct stages, static osteogenesis (SO) and dynamic osteogenesis (DO). Studies by both Ferretti et $\mathrm{al}^{28}$ and Marrotti et $\mathrm{al}^{20,29}$ concluded that $\mathrm{SO}$ is responsible for the initial layer of bone formation. As such, it is weaker woven bone but provides significant numbers of osteocyte lacunae and a stabilizing mechanism that is critical for the initial repair phase of bone healing. SO is driven by inductive stimuli and results in an increase in the bone ossification center and bone size. ${ }^{29}$ Subsequently, DO accounts for the generation of lamellar bone, resulting in bone compaction and/or thickening of the trabeculae and therefore increased mechanical integrity. The thin, bony trabeculae (10- to $15-\mu \mathrm{m}$ thick) laid down during SO provide 
the path for differentiation of osteoblast during DO. ${ }^{28}$ Dynamic osteogenesis is driven by mechanical strain sensed by osteocytes contained within the SO trabeculae. ${ }^{29}$ Therefore, the additional $15 \mu \mathrm{m}$ of expansion, equal to the thickness of trabeculae created during SO, may stimulate the osteocytes within the trabeculae to increase DO and form a thicker structure. Also, during SO osteoblasts differentiated at approximately $28 \pm 0.4 \mu \mathrm{m}$ form blood capillaries. ${ }^{5}$ Once the fusion results in a bone bridge across the interbody space, the extra $15 \mu \mathrm{m}$ of deflection from each endplate will combine to impart $30 \mu \mathrm{m}$ of compaction on the fusion mass, approximately equal to the average distance of osteoblast differentiation during SO. As such, this added compaction may also stimulate DO activity. Both of these potential methods of increased DO would result in a denser, stronger fusion.

In order to isolate the influence of the strut on the vertebral body response and endplate motion, other potential factors that influence device stiffness, such as the material type and non-endplate-contacting pores (ie, side windows), were not explored. Whereas the study confirmed increased endplate motion for a large continuous central pore, suggested to increase intracage pressure by Kanayama et $\mathrm{al},{ }^{7}$ the study outcomes are limited to the rudimentary design of the test implants. Further research should compare endplate motion with devices constructed from other clinically relevant device materials or with more complex designs such as a 3-dimensional printed porous device with a continuous or strutted central aperture.

In conclusion, endplate micromotion has been measured under periphery implant loading using a custom displacement transducer. Results demonstrate interbody cage design directly influences the motion of the vertebral endplate during dynamic loading. Dynamic cyclic subfailure loading has shown that open (or no strut) devices demonstrate an increased endplate expansion rate, and deformation into the intervertebral space when compared with a strutted design. During interbody fusion these parameters may parallel an increased rate, duration, and magnitude of applied strain on the developing fusion mass. On the basis of prior research, this type of dynamic strain on the fusion area would lead to increased bone formation and remodeling, ultimately leading to a faster and stronger interbody fusion.

\section{REFERENCES}

1. Rajaee SS, Kanim LEA, Bae HW. National trends in revision spinal fusion in the USA: patient characteristics and complications. Bone Joint J. 2014;96-B(6):807-816. https://doi. org/10.1302/0301-620X.96B6.31149

2. Smit TH, Müller R, van Dijk M, Wuisman PI. Changes in bone architecture during spinal fusion: three years follow-up and the role of cage stiffness. Spine (Phila Pa 1976). 200315;28(16):1802-1808.

3. Kandziora F, Schollmeier G, Scholz M, et al. Influence of cage design on interbody fusion in a sheep cervical spine model. J Neurosurg. 2002;96(3 Suppl):321-332.

4. Van Dijk M, Smit TH, Sugihara S, Burger EH, Wuisman PI. The effect of cage stiffness on the rate of lumbar interbody fusion: an in vivo model using Poly (L-Lactic Acid) and titanium cages. Spine (Phila Pa 1976). 2002;27(7):682-688.

5. Valdevit A, Dawoud M. Intervertebral implant performance based on dynamic stiffness response. Am J Biomed Eng. 2015;5(3):79-85. https://doi.org/10.5923/j.ajbe.20150503.01

6. Epari DR, Kandziora F, Duda GN. Stress shielding in box and cylinder cervical interbody fusion cage designs. Spine (Phila Pa 1976). 2005;30(8):908-914.

7. Kanayama M, Cunningham BW, Haggerty CJ, Abumi K, Kaneda K, McAfee PC. In vitro biomechanical investigation of the stability and stress-shielding effect of lumbar interbody fusion devices. J Neurosurg. 2000;93(2 Suppl):259-265.

8. Brinckmann $\mathrm{P}$, Frobin W, Hieholzer E, Horst $\mathrm{M}$. Deformation of the vertebral end-plate under axial loading of the spine. Spine (Phila Pa 1976). 1983;8(8):851-856.

9. Heggeness MH, Doherty BJ. Discography causes end plate deflection. Spine (Phila Pa 1976). 1993;18(8):1050-1053.

10. Ayotte DC, Ito K, Tepic S. Direction-dependent resistance to flow in the endplate of the intervertebral disc: an ex vivo study. J Orthop Res. 2001;19(6):1073-1077.

11. Setton LA, Zhu W, Weidenbaum M, Ratcliffe A, Mow VC. Compressive properties of the cartilaginous end-plate of the baboon lumbar spine. J Orthop Res. 1993;11(2):228-239.

12. Van der Veen AJ, van Dieën JH, Nadort A, Stam B, Smit $\mathrm{TH}$. Intervertebral disc recovery after dynamic or static loading in vitro: is there a role for the endplate? $J$ Biomech. 2007;40(10):2230-2235.

13. Silva MJ, Keaveny TM, Hayes WC. Load sharing between the shell and centrum in the lumbar vertebral body. Spine (Phila Pa 1976). 1997;22(2):140-150.

14. Zhang $\mathrm{F}, \mathrm{Xu} \mathrm{HC}$, Yin B, et al. Can an endplateconformed cervical cage provide a better biomechanical environment than a typical non-conformed cage? A finite element model and cadaver study. Orthop Surg. 2016;8(3):367376. https://doi.org/10.1111/os.12261.

15. Cheng CC, Ordway NR, Zhang X, Lu YM, Fang H, Fayyazi AH. Loss of cervical endplate integrity following minimal surface preparation. Spine (Phila Pa 1976). 2007;32(17):1852-1855.

16. Valdevit A, Kambic H, Elster, T, El-Said, inventors: The Cleveland Clinic Foundation, assignee. Displacement transducer. United States patent US 6,810,753 B2A. November 2, 2004.

17. Ordway NR, Rim BC, Tan R, Hickman R, Fayyazi AH. Anterior cervical interbody constructs: effect of a repetitive compressive force on the endplate. J Orthop Res. 2012;30(4):587-592. https://doi.org/10.1002/jor.21566. 
18. Valdevit A, Ullrich PF, Gallagher MB, Schneider J. Effects of cyclic loading on implant subsidence. Spine $J$. 2014;14(8):S127-S128.

19. Bauer TW, Muschler GF. Bone graft materials: an overview of the basic science. Clinical Clin Orthop Relat Res. 2000;(371):10-27.

20. Marotti, G, Zaffe D, Ferretti M, Palumbo C. Static and dynamic osteogenesis: their relevance in dental bone implants and biomaterial osseointegration. J Osteology Biomater. 2010;1(3): 133-139.

21. Lanyon LE, Rubin CT. Static versus dynamic loads as an influence on bone remodeling. J Biomech. 1984;17(12):897905.

22. Turner $\mathrm{CH}$, Forwood MR, Otter MW. Mechanotransduction in bone: do bone cells act as sensors in fluid flow? FASEB J. 1994;8(11):875-878.

23. Rubin CT, Lanyon LE. Regulation of bone formation by applied dynamic loads. J Bone Joint Surg Am. 1984;66(3):397402.

24. Duncan RL, Turner CH. Mechanotransduction and the functional response of bone to mechanical strain. Calcif Tissue Int. 1995;57(5):344-358.

25. Rubin CT, Lanyon LE. Regulation of bone mass by mechanical strain magnitude. Calcif Tissue Int. 1985;37(4):411417.

26. Frankenburg EP, Goldstein SA, Bauer TW, Harris SA, Poser RD. Biomechanical and histological evaluation of a calcium phosphate cement. J Bone Joint Surg Am. 1998;80(8):1112-1124.

27. Turner $\mathrm{CH}$, Forwood, MR, Rho J, Yoshikawa T. Mechanical loading thresholds for lamellar and woven bone formation. J Bone Miner Res. 1994;9(1):87-97.
28. Ferretti M, Palumbo C, Contri M, Marotti G. Static and dynamic osteogenesis: two different types of bone formation. Anat Embryol (Berl). 2002;206(1-2):21-29.

29. Marotti, G. Static and dynamic osteogenesis. Ital J Anat Embryol. 2010;115(1-2):123-126.

Disclosures and COl: While employed at Stevens Institute of Technology, A. Valdevit and A. Kedzierska received institutional support through a grant from Titan Spine, LLC, the manufacturer of the sample test devices. M.B. Gallagher, J.M. Schneider, and P.F. Ullrich are employees of Titan Spine, LLC.

Corresponding Author: Antonio Valdevit, $\mathrm{PhD}$, Director, Health Sciences, SEA Limited, Columbus, OH 43229. Mobile: (914) 393-7229; Office Phone: (800) 782-6851; Fax: (614) 885-8014; Email: avaldevit@sealimited.com.

Published 31 December 2019

This manuscript is generously published free of charge by ISASS, the International Society for the Advancement of Spine Surgery. Copyright (C) 2019 ISASS. To see more or order reprints or permissions, see http://ijssurgery.com. 